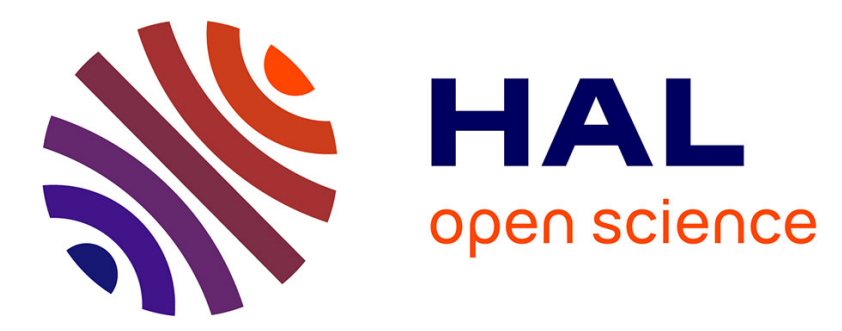

\title{
Répartition des formes d'azote et catabolisme des purines chez le dolique tubéreux, Pachyrhizus erosus Urban
}

Thierry Lamaze, Gérard Vansuyt, Claude Zinsou

\section{- To cite this version:}

Thierry Lamaze, Gérard Vansuyt, Claude Zinsou. Répartition des formes d'azote et catabolisme des purines chez le dolique tubéreux, Pachyrhizus erosus Urban. Agronomie, 1985, 5 (10), pp.933-938. hal-00884724

\section{HAL Id: hal-00884724 \\ https://hal.science/hal-00884724}

Submitted on 1 Jan 1985

HAL is a multi-disciplinary open access archive for the deposit and dissemination of scientific research documents, whether they are published or not. The documents may come from teaching and research institutions in France or abroad, or from public or private research centers.
L'archive ouverte pluridisciplinaire HAL, est destinée au dépôt et à la diffusion de documents scientifiques de niveau recherche, publiés ou non, émanant des établissements d'enseignement et de recherche français ou étrangers, des laboratoires publics ou privés. 


\title{
Répartition des formes d'azote et catabolisme des purines chez le dolique tubéreux, Pachyrhi- zus erosus Urban
}

\author{
Thierry LAMAZE $\left({ }^{*}\right)$, Gérard VANSUYT \& Claude ZINSOU \\ I.N.R.A., Laboratoire de Physiologie et Biochimie végétales, Centre de Recherches des Antilles et de la \\ Guyane, 97170 Petit-Bourg
}

RÉSUMÉ

\begin{abstract}
L'étude de l'assimilation de l'azote fixé a été entreprise chez le dolique tubéreux, Pachyrhizus erosus. Les nodosités, les limbes et les gousses renferment la majeure partie de l'azote total. L'azote soluble se trouve essentiellement dans les gousses et les tiges. La teneur en azote uréidique est très faible dans les limbes comparés aux autres organes. Les uréides présentent deux pics d'accumulation, à l'apparition des premières nodosités et au moment du remplissage des gousses. Comparés à l'asparagine, au glutamate, à la glutamine et à l'aspartate, les uréides représentent la forme biochimique de transport majeure. Quant aux enzymes, l'activité xanthine déshydrogénase n'a été détectée que dans les nodosités. L'activité uricase de ces dernières est 50 à 100 fois supérieure à celle des autres tissus. Ces deux activités montrent deux pics, l'un au début de la tubérisation, l'autre au moment du remplissage des gousses. L'activité allantoïcase, bien que détectée dans toutes les parties de la plante, semble plus importante dans les tiges. La distribution des différentes formes d'azote est discutée en relation avec l'évolution des capacités enzymatiques.
\end{abstract}

Mots clés additionnels : nodosités, uréides, xanthine déshydrogénase, uricase, allantoïcase.

Distribution of nitrogen compounds and purine catabolism in yam bean Pachyrhizus erosus Urban

Ammonia assimilation was investigated in yam bean, Pachyrhizus erosus. The nodules, leaves and pods contained the major part of total nitrogen. Soluble nitrogen was found essentially in pods and stems. Ureide nitrogen content was low in leaves as compared with other organs. The ureides showed two peaks of accumulation, at first nodule appearance and at pod development. In comparison with asparagine, glutamate, glutamine and aspartate, the ureides represented the main form of nitrogen transport. As to enzymes, xanthine dehydrogenase activity was detected only in nodules, in which uricase activity was found to be 50 to 100 times higher than in other tissues. These two enzyme activities showed two peaks, at the beginning of tuberization and at pod development. Allantoicase activity, although present in all parts of the plant, seemed to be greater in the stems. The distribution and change in content of different forms of nitrogen in the tissues is discussed in relation to changes in enzyme activity.

Additional key words : nodules, ureides, xanthine dehydrogenase, uricase, allantoicase.

\section{INTRODUCTION}

Le dolique tubéreux ou yam bean (Pachyrhizus erosus Urban), originaire de l'Amérique centrale, est une légumineuse tubérifère tropicale, de la famille des papilionacées et de la tribu des phaséolées. Le tubercule, charnu et juteux est comestible et riche en sucres directement assimilables (glucose et fructose essentiellement) (ZINSOU, trav. non publiés). Sa partie aérienne contient des quantités importantes de protéi-

(*) Adresse actuelle : I.N.R.A., Laboratoire du Métabolisme et de la Nutrition des Plantes, route de Saint-Cyr, F 78000 Versailles. nes et de lipides mais aussi de la roténone; cependant, les jeunes gousses peuvent être consommées moyennant quelques précautions de préparation (SCHROEder, 1967 ; SRIVASTAVA et al., 1973). Outre son intérêt agronomique, le dolique tubéreux, qui présente simultanément deux sites d'accumulation en concurrence pour les molécules de réserves (photosynthétats, molécules azotées...), constitue un mätériel scientifique intéressant pour l'étude de la compétition entre la tubérisation et la fructification. Les travaux de ZINSOU (non publiés) ont déjà montré que ces deux processus sont affectés par les phytohormones $\left(\mathrm{CCC}, \mathrm{GA}_{3}, \mathrm{BAP} \ldots\right)$ et sont directement dépendants 
du photopériodisme malgré les très faibles écarts entre la photophase et la nyctiphase observés en milieu tropical.

Chez certaines phaséolées d'origine tropicale comme le soja, la forme majeure de transport et de stockage de l'azote fixé est constituée par les uréides glyoxyliques (PATE et al., 1980) contrairement à d'autres espèces (lupin, trèfle, féverole...) où l'assimilation de l'azote fixé fait intervenir principalement des acides aminés et des amides (SCOTT et al., 1976 ; REYNOLDS et al., 1982). Certains auteurs considèrent que cette particularité serait une caractéristique de la tribu des phaséolées (ZENGBÉ \& SALSAC, 1983). Chez ces plantes, le métabolisme de l'azote fixé dans les nodosités commencerait par la biosynthèse de novo de purines (ATKINS et al., 1982), puis, dans ces mêmes organes, le catabolisme des bases puriques aboutirait à la formation des uréides (THOMAS \& SCHRADER, 1981). La dégradation ultérieure de ces composés dans les différentes parties de la plante permettrait la libération de l'azote incorporé lors de la synthèse des purines. Les uréides ont un rapport $\mathrm{C} / \mathrm{N}$ de 1 (JOLIVET \& MOSSÉ, 1982 ) et leur synthèse nécessite moins d'énergie que celle de l'asparagine ou de la glutamine (SCHUBERT, 1981). Ils constitueraient de ce fait une forme de transport et de stockage économique bien qu'ils ne puissent pas être directement utilisés pour la synthèse protéique.

Le dolique tubéreux, malgré l'intérêt agronomique et alimentaire qu'il peut présenter pour les régions tropicales, ne fait l'objet d'aucune recherche actuelle orientée vers la détermination de ses potentialités de fixation symbiotique de l'azote et vers la compréhension de la voie de l'assimilation de l'azote fixé. Cet article se propose d'établir l'importance respective des différents composés aminés et uréidiques qui interviennent dans le transport de l'azote fixé et présente également l'évolution des activités de 3 enzymes liées au métabolisme des uréides au cours du développement de cette plante.

\section{MATÉRIEL ET MÉTHODES}

\section{A. Matériel végétal}

Les graines de dolique tubéreux ont été aimablement fournies par le Dr STEELE de l'I.I.T.A. d'Ibadan (Nigeria) et multipliées sur place.

L'expérimentation a porté sur 250 plantes homogénéisées, issues de graines semées en juin 1984 (conditions de jours longs), tous les $60 \mathrm{~cm}$ sur des billons espacés de $1 \mathrm{~m}$. La fertilisation minérale est apportée sous forme de scories de déphosphoration et de sulfate de potassium à raison de 70 unités de $\mathrm{P}_{2} \mathrm{O}_{5}$ et de 120 unités de $\mathrm{K}_{2} \mathrm{O}$ à l'hectare. Les $2 / 3$ sont apportés au moment du semis et le reste un mois après. Aucun apport d'azote n'a été effectué. Les sols sont du type ferrallitique ; leur teneur en azote n'a pas été déterminée.

Pour les études analytiques, 3 plantes sont prélevées au hasard et conservées dans la glace jusqu'au laboratoire où elles sont lavées, essuyées puis conservées au congélateur à $-22{ }^{\circ} \mathrm{C}$.

\section{B. Dosage des uréides}

Les échantillons sont broyés à $4{ }^{\circ} \mathrm{C}$ dans $10 \mathrm{ml}$ de tampon Tris $\mathrm{HCl} 0,05 \mathrm{M}, \mathrm{pH} 7,5$ contenant $0,05 \mathrm{~g}$ de polyclar AT par g de tissus frais (MATSUMOTO et al., $1977 a$ ). Après centrifugation à $6000 \mathrm{~g}$ pendant $45 \mathrm{mn}$, une partie aliquote du surnageant est utilisée pour le dosage des uréides (allantoïne + acide allantoïque) selon la méthode de YOUNG \& CONWAY (1942).

\section{Dosage de l'azote total et de l'azote soluble}

L'azote total a été dosé après minéralisation selon la méthode Kjeldahl. L'ammonium produit est déplacé par la soude $12 \mathrm{~N}$ et titré par $\mathrm{H}_{2} \mathrm{SO}_{4} 0,1 \mathrm{~N}$ en présence de l'indicateur Tashiro. L'azote soluble a été déterminé sur $2 \mathrm{~g}$ de tissus séchés à $60^{\circ} \mathrm{C}$. L'extraction a été réalisée par le mélange éthanol/eau : 50/50 à $80^{\circ} \mathrm{C}$ selon la technique de LefEBvRE (1958). Dans l'extrait sont dosés l'azote ammoniacal, l'azote nitrique et l'azote organique après précipitation des protéines par l'acide trichloracétique (20 p. 100). (Déterminations effectuées par le Laboratoire d'Analyses de la Station d'Agronomie, Petit-Bourg.)

\section{Extraction et analyse des acides aminés et des amines}

Sur des tissus pesés et congelés, les extractions sont effectuées par un mélange éthanol/eau : 80/20 renfermant 1 p. 100 d'acide acétique glacial suivant la technique de VALLÉE et al. (1983). Les analyses séquentielles des acides aminés et des amines sont effectuées au moyen d'un autoanalyseur Technicon selon une technique basée sur la méthode de MOORE \& STEIN (1954) pour les acides aminés et selon la technique de VILlANUEVA et al. (1977) pour les amines. Les dosages sont effectués au moyen de la réaction à la ninhydrine à $570 \mathrm{~nm}$.

L'évaluation des teneurs en asparagine et glutamine est obtenue par la méthode de VALléE (1973). Les dosages d'acide aspartique (Asp) et glutamique (Glu) sont effectués sur un même échantillon, avant et après hydrolyse par $\mathrm{HCl} 2 \mathrm{~N}$ pendant $4 \mathrm{~h}$ à $100^{\circ} \mathrm{C}$. L'hydrolysat est ramené à $\mathrm{pH}$ 2,0 avec une quantité connue de soude avant la $2^{\mathrm{e}}$ analyse. La différence entre les valeurs obtenues pour Asp et Glu avant et après hydrolyse nous donne les teneurs en asparagine et en glutamine.

\section{E. Mesure des activités enzymatiques}

Les échantillons ( $1 \mathrm{~g}$ de tissu frais) sont broyés à $4^{\circ} \mathrm{C}$ dans $5 \mathrm{ml}$ de tampon Tris $\mathrm{HCl} 0,1 \mathrm{M}, \mathrm{pH} 7,5$, contenant du dithiothréitol $1 \mathrm{mM}$ et de l'EDTA $1 \mathrm{mM}$ (HERRIDGE et al., 1978). Après centrifugation à $6000 \mathrm{~g}$ pendant $45 \mathrm{mn}$, une fraction aliquote du surnageant subit une perméation sur une hauteur de $15 \mathrm{~cm}$ de gel Sephadex G 25 dans une colonne thermostatée à $4{ }^{\circ} \mathrm{C}$, de $2,5 \mathrm{~cm}$ de diamètre. L'élution se fait à l'aide du tampon Tris $\mathrm{HCl} 20 \mathrm{mM}(\mathrm{pH} 7,5)$. Les protéines, exclues du gel et détectées à $280 \mathrm{~nm}$, constituent l'extrait enzymatique. 
La réaction enzymatique est réalisée à $35^{\circ} \mathrm{C}$. Les activités $\mathrm{XDH}(\mathrm{EC}$ 1.2.1.37), uricase (EC 1.7.3.3) et allantoïcase (EC 3.5.3.4) ont été déterminées selon ZENGBÉ \& SALSAC (1983). L'activité allantoïnase n'a pas été déterminée car l'enzyme contrôlant l'étape ultime de la dégradation des uréides et donc l'utilisation de l'azote stocké sous cette forme biochimique est l'allantoïcase. Ce choix nous semble aussi justifié par le fait que plus de 80 p. 100 de l'azote uréidique est sous forme d'allantoate (résultat non présenté). Les protéines sont dosées sur l'extrait utilisé pour les dosages enzymatiques par la méthode de BRADFORD (1976). Les résultats sont la moyenne de 2 ou 3 répétitions au minimum.

\section{RÉSULTATS}

Au cours du cycle de développement de la plante, la teneur en azote total des tiges et surtout du tubercule est inférieure à celle des nodosités, des limbes et des gousses (fig. 1). Les niveaux d'azote soluble, mesurés sur l'ensemble du cycle et exprimés en p. 100 de l'azote total, sont plus élevés dans les gousses, puis dans les tiges, et plus bas dans les nodosités et les limbes (tabl. 1).

La teneur en azote uréidique des limbes (fig. $2 \mathrm{C}$ ) est très faible comparée à celle des autres organes (fig. 2 A, B et D). Dans les nodosités et les gousses, cette teneur diminue au cours du cycle de végétation. Dans les tiges, les quantités d'uréides passent par un maximum après l'apparition des $1^{\text {res }}$ nodosités et une nouvelle accumulation a lieu lors du remplissage des gousses (fig. 2 B et D). Les quantités d'uréides obtenues dans les hampes florales sont importantes et du

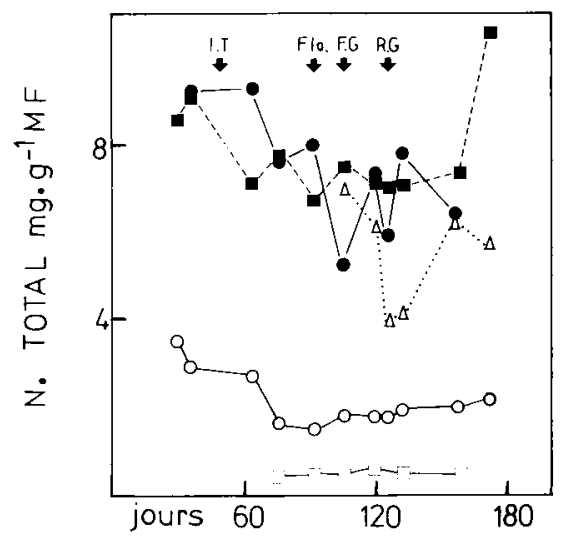

Figure 1

Evolution de la teneur en azote total dans les différents tissus au cours du développement du dolique tubéreux : limbes (ם), tiges ( )), tubercules $(\square)$, nodosités $(\bullet)$, gousses $(\Delta)$. Les flèches indiquent respectivement: IT, induction de la tubérisation; Flo, floraison; F-G, formation des gousses ; R.G., remplissage des gousses. Change in total nitrogen content in different tissues during development of yam bean : leaves ( $\mathbf{\square})$, stems $(0)$, tubers $(\square)$, nodules $(\bullet)$, pods $(\Delta)$. The abbreviations stand for: IT, induction of tuberization ; Flo, flowering ; F-G, pod formation ; R.G., pod development.

même ordre de grandeur que celles observées dans les gousses ; ces composés sont difficilement détectables dans le tubercule (résultats non publiés).

Mesurés sur l'ensemble du cycle végétatif et comparés à l'asparagine, glutamate, glutamine et aspartate qui sont considérés comme des formes de transport de l'azote fixé, les uréides représentent la forme biochimique azotée majeure.

TABLEAU 1

Composition moyenne des tissus en différentes formes d'azote calculée sur l'ensemble du cycle du dolique tubéreux. L'azote est exprimé en mg ou en pour cent de l'azote total ou soluble. Average content of different forms of nitrogen in different tissues calculated over the whole cycle of yam bean. Nitrogen expressed in $\mathrm{mg}$ or in percent of total nitrogen.

\begin{tabular}{|c|c|c|c|c|c|c|c|c|}
\hline \multirow{2}{*}{$\mathbf{N}$} & \multicolumn{2}{|c|}{ Nodosités } & \multicolumn{2}{|c|}{ Tiges } & \multicolumn{2}{|c|}{ Limbes } & \multicolumn{2}{|c|}{ Gousses } \\
\hline & $\mathrm{mg}$ & $\%$ & $\mathrm{mg}$ & $\%$ & $\mathrm{mg}$ & $\%$ & $\mathrm{mg}$ & $\%$ \\
\hline Total & 7,43 & & 2,17 & & 7,80 & & 5,59 & \\
\hline Soluble & 1,76 & 23,7 & 0,64 & 29,4 & 1,92 & 24,6 & 1,98 & 35,4 \\
\hline Protéines solubles & 0,68 & 38,6 & 0,20 & 31,2 & 0,99 & 51,5 & 0,71 & 35,8 \\
\hline Aminés libres & 0,67 & 38,0 & 0,29 & 45,8 & 0,47 & 24,4 & 0,73 & 37,1 \\
\hline$\underset{\text { Glu }}{\text { Asp }}+\underset{\text { Gln }}{\text { Asn }}$ & 0,26 & 14,6 & 0,12 & 19,5 & 0,17 & 9,1 & 0,34 & 17,4 \\
\hline Uréides & 0,21 & 12,2 & 0,11 & 17,8 & 0,02 & 1,2 & 0,41 & 20,6 \\
\hline$a / b$ & \multicolumn{2}{|c|}{1,2} & \multicolumn{2}{|c|}{1,9} & \multicolumn{2}{|c|}{7,29} & \multicolumn{2}{|c|}{0,84} \\
\hline Asparagine & 0,034 & 1,9 & 0,056 & 8,8 & 0,022 & 1,1 & 0,157 & 7,9 \\
\hline Aspartique & 0,030 & 1,7 & 0,012 & 1,9 & 0,013 & 0,7 & 0,028 & 1,4 \\
\hline Glutamine & 0,118 & 6,7 & 0,017 & 2,7 & 0,067 & 3,5 & 0,068 & 3,4 \\
\hline Glutamique & 0,076 & 4,3 & 0,040 & 6,3 & 0,073 & 3,8 & 0,093 & 4,7 \\
\hline
\end{tabular}



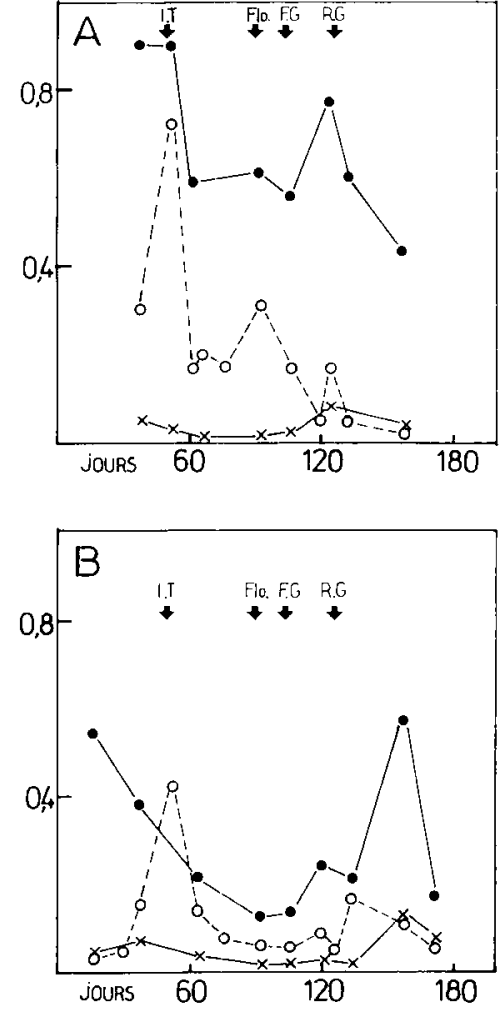

Figure 2

Evolution des différentes formes d'azote dans les différents tissus. $\mathbf{A}$, nodosités ; $\mathbf{B}$, tiges ; $\mathbf{C}$, limbes et $\mathbf{D}$ gousses : $\mathbf{N}$ aminés libres $(\bullet), \mathbf{N}$ uréides (O); $\mathbf{N}$ asparagine $(x)$.

L'analyse des amines aliphatiques a révélé des quantités importantes de putrescine dans les gousses au moment de leur formation ainsi que dans les nodosités durant la même période. Ces teneurs sont de l'ordre de 2 p. 100 des composés aminés libres. La putrescine s'accumule dans le limbe et les tiges lors du remplissage des graines jusqu'à représenter respectivement 1 et 0,5 p. 100 des composés aminés libres. De faibles quantités de spermidine ont été détectées dans tous les organes.

La xanthine déshydrogénase, l'uricase et l'allantö̈case sont des enzymes qui interviennent dans le catabolisme des purines. Les 2 premières catalysent les étapes biochimiques conduisant à la synthèse des uréides, alors que le dernier contrôle leur dégradation. Dans nos conditions expérimentales, l'activité xanthine déshydrogénase n'a été détectée que dans les nodosités et, dans ces tissus, l'activité uricase est 100 fois plus importante que dans les autres (fig. $3 \mathrm{~A}$ et $B$ ). Ces activités montrent un maximum entre le début de la tubérisation et l'apparition des $1^{\text {res }}$ gousses, la capacité catalytique des 2 enzymes diminue ensuite d'une manière similaire (fig. $3 \mathrm{~A}$ ). Une très faible activité allantoïcase a été mesurée dans toutes les parties de la plante. Les tiges semblent présenter une capacité enzymatique plus importante mais cette activité n'est, en moyenne, que 3 fois supérieure à celle observée dans les nodosités (fig. 3 A et B). Les quantités de protéines solubles contenues dans les tiges sont de 3 à 5 fois inférieures à celles des autres organes (tabl. 1). Il en résulterait une activité allantö̈case spécifique plus importante dans cette partie de la plante.
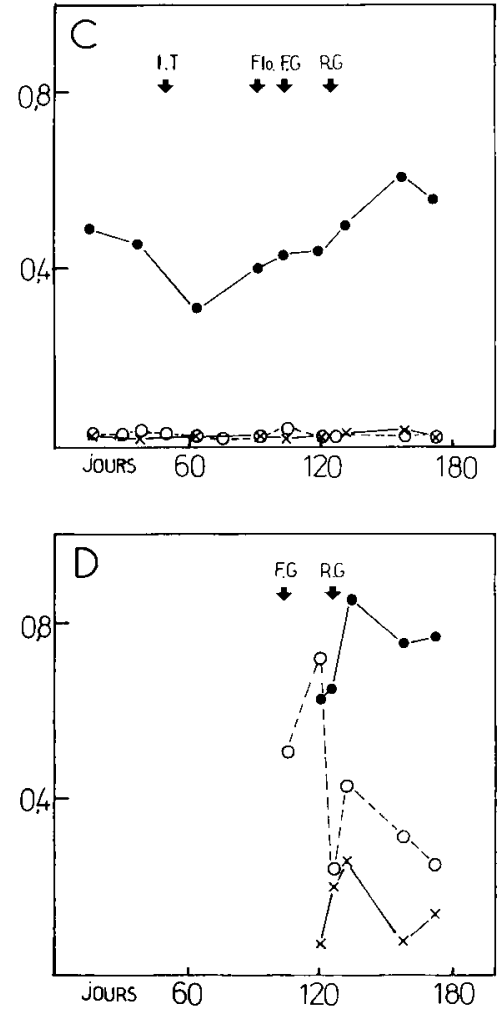

Change in different nitrogen forms in tissues. $\mathbf{A}$, nodules; $\mathbf{B}$, stems ; $\mathbf{C}$, leaves ; $\mathbf{D}$, pods : $\mathbf{N}$ free aminoacids $(\bullet), \mathbf{N}$ ureides $(\bigcirc)$, $\mathrm{N}$ asparagine $(x)$.

\section{DISCUSSION}

En milieu tropical humide, les rendements totaux du dolique tubéreux sont compris entre 60 et $120 \mathrm{t}$ de tubercules par ha (ZINSOU, comm. pers., 1985) et les sols de culture ferrallitique, non amendés, contiennent une faible quantité d'azote (DUCHAUFOUR, 1977). Nos parcelles expérimentales n'ont reçu aucune fumure autre que potassique et phosphorique; il en résulte qu'une grande part de l'azote contenu dans la plante doit provenir de la fixation symbiotique qui s'avère efficace.

Dans les nodosités, les tiges et les gousses, l'azote sous forme uréidique constitue une partie importante de l'azote soluble (tabl. 1). Néanmoins, la quantité d'azote représentée par l'ensemble des autres composés aminés libres est 2 à 3 fois supérieure à celle de l'azote uréidique. Chez le soja, les teneurs en uréides des organes diminuent lorsque la plante est alimentée avec $\mathrm{NO}_{3}^{-}$(MATSUMOTO et al., 1977b) et la contribution de l'azote nitrique à la formation des uréides est faible (OHYAMA et al., 1981). De même lorsque les racines sont autorisées à absorber $\mathrm{NH}_{4}^{+}$, ce dernier se retrouve principalement sous forme d'acides aminés (FUJIHARA \& YAMAGUCHI, 1978). Nos conditions expérimentales sont celles de la plante au champ et, bien que la quantité d'azote nitrique ou ammoniacale du sol soit certainement faible, sa contribution à l'alimentation du dolique tubéreux pourrait expliquer l'importance de l'azote sous forme d'amines libres contenu dans la plante. Dans ces conditions, nos résultats paraissent confirmer l'hypothèse selon laquelle une des formes prédominantes de transport et 


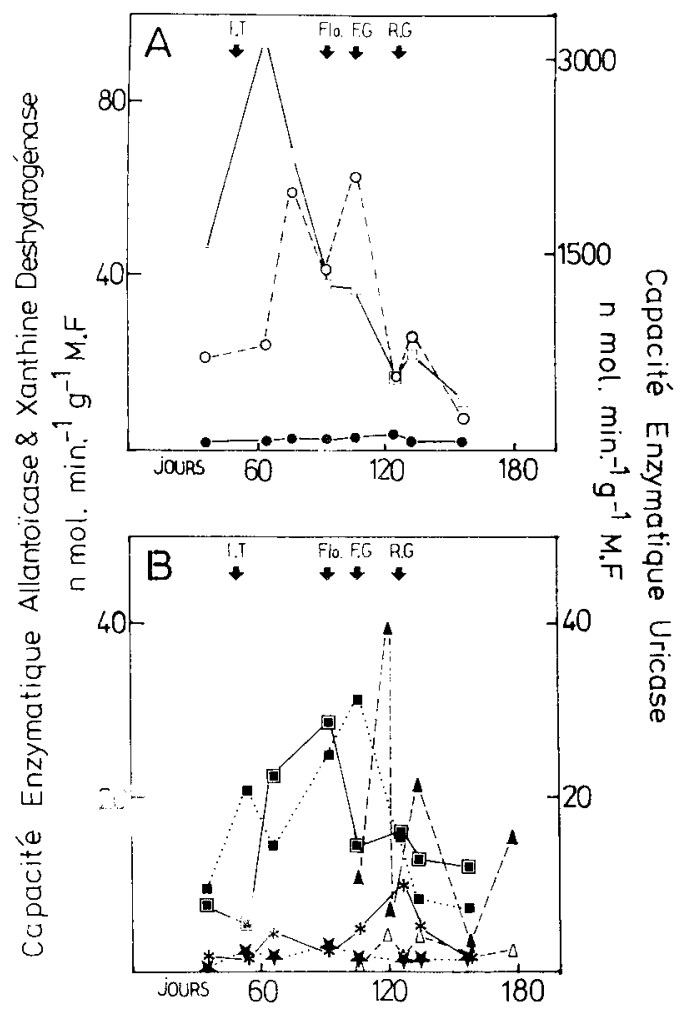

Figure 3

Evolution des activités enzymatiques, allantoïcase, xanthine déshydrogénase et uricase. A nodosités : uricase ( $\square$ ) ; xanthine déshydrogenase (O); allantoïcase (๑). B activité allantoicase : tige (*); feuille ( $\star$ ); gousse $(\Delta)$. Activité uricase : tige ([) ; feuille (ם); gousse $(\mathbf{\Delta})$.

Change in allantoicase, xanthine dehydrogenase and uricase activities. A nodules : uricases $\square$ ); xanthine dehydrogenase (O); allan-

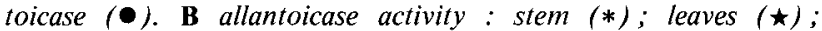
pods $(\Delta)$. Uricase activity : stem (回); leaves $(\mathbf{\square})$; pods $(\boldsymbol{\Delta})$.

de stockage de l'azote fixé, chez les phaséolées, serait constituée par les uréides (MC CLURE \& ISRAEL, 1979 ; STREETER, 1979; MC CLURE et al., 1980 ; ZENGBÉ \& SALSAC, 1983). Chez le soja, la synthèse de ces molécules se situerait au niveau des nodosités et impliquerait le catabolisme des bases puriques (TAJIMA \& YAMAMOTO, 1975 ; TAJIMA et al., 1977 ; MATSUMOTO et al., 1977 ; ZENGBÉ \& SALSAC, 1983). Chez le dolique tubéreux, les activités de la xanthine déshydrogénase et de l'uricase ne sont importantes que dans les nodosités (fig. 3). Ces 2 enzymes catalysent des réactions conduisant à la formation des uréides selon la voie de dégradation des purines. Dans ces conditions, il est probable que les nodosités sont le lieu privilégié de la synthèse de ces molécules qưi peuvent par ailleurs s'y accumuler. Une activité allantoïcase a été mise en évidence dans ces organes. Il se pourrait donc qu'une partie des uréides soit dégradée sur leur lieu de formation contribuant ainsi à l'apparition des autres composés aminés libres.

La détermination des formes biochimiques de transport de l'azote depuis les nodosités jusqu'aux différents organes et l'étude de leur utilisation par la plante sont souvent basées sur des analyses de sève xylémique et/ou sur le marquage au ${ }^{15} \mathrm{~N}$ (OHYAMA \& KumaZAWA, 1979 ; STREETER, 1979 ; COOKSON et al., 1980). De telles mesures n'ont pas pu être effec- tuées. Cependant, la capacité enzymatique de l'allantoïcase relevée dans les tiges et les limbes (fig. 3) et les teneurs en uréides de ces organes (fig. 2) laissent supposer que la dégradation de ces composés a lieu avant ou au cours de leur transfert dans les limbes. Dans ces organes, les formes azotées issues de la dégradation de l'acide allantoïque et des biosynthèses ultérieures viennent s'ajouter aux composés aminés solubles. L'ensemble des quantités en aspartate, asparagine, glutamate et glutamine devient alors largement dominant comparé aux uréides (tabl. $1 \mathrm{a} / \mathrm{b})$. Ainsi la forme azotée majeure issue de la fixation serait transformée avant d'être distribuée dans les feuilles et utilisée pour la synthèse protéique ou le métabolisme des composés aminés notamment.

Une autre hypothèse peut être avancée : de récents travaux, utilisant le marquage au ${ }^{15} \mathrm{~N}$ (YONEYAMA \& ISHIZUKA, 1982 ; WAREMBOURG \& FERNANDEZ, 1985) ont montré que, chez le soja, l'azote provenant de la fixation symbiotique était dirigé préférentiellement vers les organes en croissance. Cette discrimination, si elle était réalisée chez le dolique tubéreux, pourrait être à l'origine des faibles teneurs en uréides observées dans les feuilles et de l'accumulation de ces composés dans les gousses.

L'activité allantoïcase mesurée chez cette plante est faible dans tous les tissus y compris dans la tige. Pourtant les parties aériennes, compte tenu de leur masse importante (4 à $5 \mathrm{~kg}$ de matière frâiche par plante) ont certainement une activité totale suffisante pour assurer la dégradation de tout l'acide allantoïque présent dans la plante et qui doit être nettement supérieure à la capacité catalytique des nodosités $(100 \mathrm{~g}$ de matière fraîche par plante).

Chez les légumineuses, les gousses constituent un site de forte accumulation pour beaucoup de biomolécules mais surtout pour les composés azotés en vue de l'élaboration des réserves de la graine. Les uréides semblent y jouer un rôle important puisqu'il constitue en moyenne 55 p. 100 de l'azote «aminé libre» (tabl. $1 \mathrm{a} / \mathrm{b}$ ). Cependant ce rapport va en diminuant au cours de la maturation des gousses. Les uréides ne seraient qu'une forme provisoire de stockage de l'azote contenue dans les fruits du Pachyrhizus erosus.

Ce premier travail a permis de déterminer la forme biochimique majeure de transport de l'azote fixé. Les disponibilités et la répartition des composés aminés et uréidiques diffèrent selon les tissus. L'étude a été menée en jours longs où le développement de la partie aérienne est prépondérant alors qu'en jours courts c'est celui de la partie souterraine qui est privilégié. Elle va être poursuivie en jours courts de manière à établir les effets du climat sur la fixation symbiotique, l'assimilation de l'azote et le métabolisme des glucides chez cette légumineuse tubérifère.

Reçu le 13 mai 1985. Accepté le 30 juillet 1985.

\section{REMERCIEMENTS}

Nous tenons à exprimer nos sincères remerciements à Mme Sobesky O. du Laboratoire d'Analyses de la Station d'Agronomie pour le dosage des azotes ammoniacal, nitrique et organique. 


\section{RÉFÉRENCES BIBLIOGRAPHIQUES}

Atkins C. A., Ritchie A., Rowe P. B., Mc Cairns E., Sauer D., 1982. De novo purine synthesis in nitrogen-fixing nodules of cowpea (Vigna unguiculata (L.) Walp.) and soybean (Glycine max (L.) Merr.). Plant Physiol., 70, 55-60.

Bradford M. M., 1976. A rapid and sensitive method for the quantitation of microgram quantities of protein utilizing the principle of protein-dye binding. Anal. Biochem., 72, 248-254.

Cookson C., Hugues H., Coombs J., 1980. Effects of combined nitrogen on anaplerotic carbon assimilation and bleeding sap composition in Phaseolus vulgaris L., Planta, 148, 338-345.

Duchaufour P., 1977. Pédogenèse et classification, 438. In $\mathrm{Ph}$ Duchaufour \& B. Souchier : “Pédologie », Masson, Paris.

Fujihara S., Yamaguchi M., 1978. Probable site of allantoin formation in nodulating soybean plants. Phytochemistry, 17, 1239-1243.

Herridge D. F., Atkins C. A., Pate J. S., Rainbird R. M., 1978. Allantoin and allantoic acid in the nitrogen economy of the cowpea (Vigna unguiculata (L.) Walp.). Plant Physiol., 62, 495-498.

Jolivet E., Mossé J., 1982. Non-protein nitrogenous compounds with particular attention to ureides, 195-216. In Aroba S. K. : «Chemistry and biochemistry of legumes ». Oxford IBH Publ. Co, New Delhi.

Lefebvre J. M., 1958. Etude des variations des substances azotées au cours de la végétation de l'orge. Thèse Doct. Univ. Paris.

Matsumoto T., Yatazawa M., Yamamoto Y., 1977a. Distribution and change in the content of allantoin and allantoic acid in developing nodulating and non nodulating soya bean plants. Plant Cell Physiol., 18, 353-359.

Matsumoto T., Yatazawa M., Yamamoto Y., 1977b. Effects of exogenous nitrogen compounds on the concentrations of allantoin and various constituents in several organs of soybean plants. Plant Cell Physiol., 18, 613-624.

Mc Clure P. R., Israel D. W., 1979. Transport of nitrogen in xylem of soybean plants. Plant Physiol, 64, 411-416.

Mc Clure P. R., Israel D. W., Volk R. J., 1980. Evaluation of the relative ureide content of xylem sap as indicator of $\mathrm{N}_{2}$ fixation in soybeans. Green house studies. Plant Physiol., 66, 720-725.

Moore S., Stein W., 1954. Procedures for the chromatographic determination of amino acids on $4 \%$ cross-linked sulfonated polystyrene resins. J. Biol. Chem., 211, 893-913.

Ohyama T., Kumazawa K., 1979. Assimilation and transport of nitrogenous compounds originated from ${ }^{15} \mathrm{~N}_{2}$ fixation and ${ }^{15} \mathrm{NO}_{3}$ assimilation. Soil Sci. Plant Nutr., 25, 9-19.

Ohyama T., Owa N., Fujishima Y., Kumazawa K., 1981. Nitrogen assimilation in soybean nodules. IV. Allantoin formation and transport in relation to supply with various forms of combined nitrogen. Soil Sci. Plant Nutr., 27, 55-64.

Pate J. S., Atkins C. A., White R. T., Rainbird R. S., Woo K. C.,
1980. Nitrogen nutrition and xylem transport of nitrogen in ureideproducing grain legumes. Plant Physiol., 65, 961-965.

Reynolds P. H. S., Blevins D. G., Boland H. J., Schubert K. R., Randall D. D., 1982. Enzymes of ammonia assimilation in legume nodule : a comparison between ureide - and amide - transporting plants. Physiol. Plant., 55, 255-260.

Schubert K. R., 1981. Enzymes of purine biosynthesis and catabolism in Glycine max. I. Comparison of activities with $\mathrm{N}_{2}$ fixation and composition of xylem exudate during nodule development. Plant Physiol., 68, 1115-1122.

Schroeder C. A., 1967. The jicama, a rooterop from Mexico. Ann. Soc. Hortic. Sci., 11, 65-71.

Scott D. B., Farnden K. J. K., Robertson J. G., 1976. Ammonia assimilation in lupin nodules. Nature, 263, 703-705.

Srivastava G. S., Shukla D. S., Awaasthi D. N., 1973. We can grow sankalu in the plains of Uttar Pradesh. Indian Farming, 33, 39.

Streeter J. G., 1979. Allantoin and allantoic acid in tissues and stem exudate from field-grown soybean plants. Plant Physiol., 63, 478-480.

Tajima S., Yamamoto $Y_{.,}$1975. Enzymes of purine catabolism in soybean plants. Plant Cell. Physiol., 16, 271-282.

Tajima S., Yatazawa M., Yamamoto Y., 1977. Allantoin production and its utilization in relation to nodule formation in soybeans : enzymatic studies. Soil Sci. Plant Nutrit., 23, 225-235.

Thomas R. J., Schrader L. E., 1981. Ureide metabolism in higher plants. Phytochemistry, 20, 361-371.

Vallée J. C., 1973. Quelques aspects du métabolisme de la proline chez divers Nicotiana en fonction du développement. Thèse Doct. Etat, Fac. Sci. de Dijon.

Vallée J. C., Vansuyt G., Negrel J., Negrel J., Perdrizet E., Prévost J., 1983. Mise en évidence d'amines liées à des structures cellulaires chez Nicotiana tabacum et Lycopersicum esculentum. Physiol. Plant., 57, 143-148.

Villanueva V. R., Adlka R. C., Cantera-Soler A. M., 1977. Détermination rapide des polyamines et de quelques mono et diamines dans les extraits végétaux. $J$. Chromatogr., 139, 381-385.

Warembourg F. R., Fernandez H. P., 1985. Distribution et remobilisation de l'azote d'origine symbiotique. Physiol. Plant. (sous presse).

Yoneyama T., Ishizuka J., $1982 .{ }^{15} \mathrm{~N}$ study on the partitioning of the nitrogen taken by soybeans from atmospheric dinitrogen, medium nitrate or ammonium. Soil Sci. Nutr., 28, 451-461.

Young E. G., Conway C. F., 1942. On the estimation of allantoin by the Rimini-Schryver reaction. J. Biol. Chem., 142, 839-853.

Zengbé M. Salsac L., 1983. Variation des teneurs en uréides et en enzymes du catabolisme purique chez Glycine max. Physiol. Vég., 21, 67-76. 